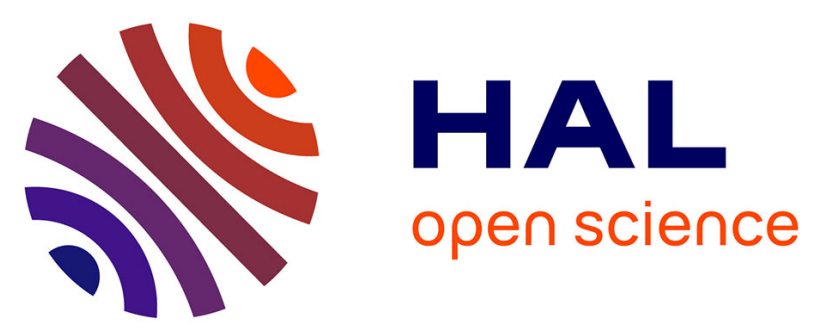

\title{
Distinctive Low-Resolution Structural Features of Dimers of Antibody Drug Conjugates and Parent Antibody determined by Small-Angle X-Ray Scattering
}

Didier Law-Hine, Sergii Rudiuk, Audrey Bonestebe, Romain Ienco, Sylvain Huille, Christophe C. Tribet

\section{To cite this version:}

Didier Law-Hine, Sergii Rudiuk, Audrey Bonestebe, Romain Ienco, Sylvain Huille, et al.. Distinctive Low-Resolution Structural Features of Dimers of Antibody Drug Conjugates and Parent Antibody determined by Small-Angle X-Ray Scattering. Molecular Pharmaceutics, 2019, 16 (12), 10.1021/acs.molpharmaceut.9b00792 . hal-02536489

\section{HAL Id: hal-02536489 \\ https://hal.science/hal-02536489}

Submitted on 8 Apr 2020

HAL is a multi-disciplinary open access archive for the deposit and dissemination of scientific research documents, whether they are published or not. The documents may come from teaching and research institutions in France or abroad, or from public or private research centers.
L'archive ouverte pluridisciplinaire HAL, est destinée au dépôt et à la diffusion de documents scientifiques de niveau recherche, publiés ou non, émanant des établissements d'enseignement et de recherche français ou étrangers, des laboratoires publics ou privés. 


\section{Distinctive Low-Resolution Structural Features of}

\section{Dimers of Antibody Drug Conjugates and Parent}

\section{Antibody determined by Small-Angle X-Ray \\ Scattering}

Didier Law-Hine ${ }^{a}{ }^{*}$, Sergii Rudiuk $^{a}$, Audrey Bonestebe ${ }^{b}$, Romain Ienco $^{b}$, Sylvain Huille ${ }^{b}$ and Christophe Tribet ${ }^{a}$ *.

${ }^{a}$ Département de Chimie, PASTEUR, École Normale Supérieure, PSL University, Sorbonne Université, CNRS, Paris, 75005, France

${ }^{\mathrm{b}}$ Biologics Formulation \& Process Development, Biologics Development, SANOFI R\&D, 13 quai Jules Guesde- BP 14, Vitry-sur-Seine, 94403, France.

KEYWORDS: Antibody-drug conjugates; size exclusion small-angle X-ray scattering (SECSAXS); aggregation; ab initio and rigid body modelling. 


\section{ABSTRACT}

Structural features of lysine-conjugated an antibody-drug conjugate (ADC) from humanized IgG1 were studied by small-angle X-ray scattering (SAXS). As the physicochemical properties of the cytotoxic drug (payload) and linker may impact the conformational and colloidal stability of the conjugated $\mathrm{mAb}$, it is essential to characterize how the conjugation may affect the overall higher order structure and therefore the physical stability and integrity of the ADCs upon storage conditions. Here, ADC monomer and aggregates generated upon thermal stress were analyzed by HPLC coupled to SAXS with a particular focus on the fraction of dimers $\left(3 \%-10 \%\right.$ depending on the storage conditions at $25^{\circ} \mathrm{C}$ and $\left.40^{\circ} \mathrm{C}\right)$. In addition to average parameters such as radius of gyration, molecular weight, maximal end-to-end distance, the structural information obtained from SAXS patterns were visualized as a low-resolution average envelope of both monomers and dimers (implementation of two methods: $a b$ initio reconstruction or modelling Fab and $\mathrm{Fc}$ as rigid bodies with a flexible hinge). We show that the monomer envelope of the ADC was similar to the corresponding (non-conjugated) parent monoclonal antibody $(\mathrm{mAb})$. ADC dimers appeared more compact and less polydisperse than the dimers of mAb, which was also confirmed by AFM. The generated envelopes of the mAb dimers suggest elongated structures with one or few inter-mAb contacts at the outermost region of Fab or Fc domains. The structural features of ADC dimers are independent of the tested $\mathrm{pH}$ buffering system (pH5.0/acetate and $\mathrm{pH} 6.0$ /histidine with or without $\mathrm{NaCl}$ ), and characterized by multiple, tighter contacts between the Fab and Fc domains, and distortion of the monomer native shape. Conjugation of a hydrophobic drug thus appeared to favor innermost inter-ADC contacts in the dimer which differed from the inter-mAb ones. 


\section{Introduction}

As the most abundant blood-circulating human antibody subclass, IgG1 has been extensively studied and is predominant among antibody scaffolds that are exploited for developments of new biotherapeutics, such as antibody drug conjugates (ADC). Artificial modifications introduced in therapeutic monoclonal antibody $(\mathrm{mAb})$ and $\mathrm{ADC}$ can however affect their stability, giving rise to irreversible aggregation and corollary adverse effects such as toxicologic and immunologic responses ${ }^{1}$. Accordingly, prevention of ADC aggregation in solutions is a major challenge hampering the fast growth of this new class of drugs ${ }^{2}$. Debates on ADC stability issues mention multiple origins of the destabilization and irreversible oligomerization. They are either rooted in properties of the mAb scaffold (high sensitivity to modifications of the mAb solubility and folding, ${ }^{3}$ or are specific to conjugation patterns and chemistry, such as hydrophobic binding of the payload, ${ }^{4}$ presence of unstable species due to heterogeneity of conjugation, ${ }^{5-7}$ chemical degradation or reactivity of the conjugated moieties. ${ }^{8}$ Knowing whether the inter-protein association predominantly comes from the mAb scaffold or from the conjugated moieties can help and fasten the improvement of potent antibodies, by focusing the efforts on the main source of instability (for instance to design more homogeneous conjugation patterns). ${ }^{9}$ Common experimental characterizations of instability, relying on measurements of aggregation rate and/or of mean inter-protein interaction in solution, do not enable direct identification of the involvement of conjugated moieties in ADC-ADC irreversible binding. In contrast, structurerelated information can help to discriminate whether or not similar domains or areas are involved in inter-mAb and inter-ADC binding. We used here robust, small angle X-ray scattering (SAXS) characterization methods and low-resolution analysis to collect structural information on ADC 
soluble aggregates, with the aim to assess the impact of formulation buffer on inter-ADC (irreversible) contacts and to compare these structural features with that obtained on unmodified $\mathrm{mAb}$ oligomers.

The recent advent of ab initio structural reconstructions from small-angle X-ray and/or neutron scattering (SAXS/SANS) of protein solutions provide now the envelopes of complex protein shapes at $\sim \mathrm{nm}$ resolution, with no need for crystallization. ${ }^{10,11}$ Although atomic resolution may not be reached, the approach is becoming a powerful tool to address questions on protein plasticity and shape variations as a function of buffer conditions, ${ }^{12}$ or protein oligomerization. ${ }^{13}$ In the specific case of antibodies, Svergun and coll. ${ }^{14,15}$ were pioneering $a b$ initio shape reconstruction when they examined solution conformation of IgG2 showing that a predominant T-shape is preserved in the $4-8 \mathrm{pH}$ window. Recent developments combine computer-generated ensembles of mAb conformations with SAXS data analysis to explore the impact of osmolytes on conformational equilibrium. ${ }^{16}$ Finally, SAXS provides the appropriate resolution window to characterize the impact of stress on $\operatorname{IgG}$ stability at molecular (shape change) and supra molecular levels (e.g, clustering ${ }^{17}$ or transient dimer formation ${ }^{18}$ ). To our knowledge, low resolution structures of ADC clusters are however not reported yet. Here we analyzed the SAXS structural features of monomers of an ADC and irreversible dimers formed during storage in different $\mathrm{pH}$ buffering systems. Ageing was accelerated by storage in thermal stress conditions at $25^{\circ} \mathrm{C}$ or $40^{\circ} \mathrm{C}$ for up to 48 days. The soluble aggregates were fractionated and analyzed with HPLC coupled to SAXS (SEC-SAXS) to collect information on the monomer, the dimers (whose dispersity was in addition imaged by AFM), and up to oligomers and high molecular weight species (HMW). The average extension/compactness, and low-resolution 
envelopes of ADC monomers and dimers were examined and compared with those of the $\mathrm{mAb}$ sharing the same primary sequence.

\section{Experimental Section}

Unless otherwise specified, chemicals were purchased from Sigma Aldrich.

Preparation of the samples. The monoclonal human $\mathrm{IgG1}$ antibody $(\mathrm{mAb})$ and the antibody drug conjugates (ADC) sharing an identical sequence were obtained from SANOFI. Both were formulated as stock solutions in $5 \mathrm{mM}$ acetic acid-sodium acetate pH 5.0 buffer (named "NaAc" buffer) at ca. $30 \mathrm{~g} / \mathrm{L}$ and stored at $-80^{\circ} \mathrm{C}$. Two other dilution buffers made of 1) $20 \mathrm{mM}$ acetic acid-sodium acetate and $136 \mathrm{mM}$ sodium chloride $\mathrm{pH} 5.0$ ("NaCl" buffer) or 2) $20 \mathrm{mM}$ histidine$\mathrm{HCl}$ pH 6.0 ("His" buffer) were used for the experiments. The ADC was obtained in two steps by stochastic conjugation of the mAb on lysines with an hydrophobic drug ${ }^{7,19}$ (the first step was the coupling of N-hydroxysuccinime-actived linkers containing an oligo(ethyleneglycol) strand and the second step was a thiol-disulfide exchange onto the other end of the mAb-attached linkers). This procedure yields a population of conjugated molecules with an average drug:IgG molar ratio of 3.9:1, with stochastic distribution of the conjugation points. To prepare samples, aliquots of $500 \mu \mathrm{L}$ of the stock solutions (in NaAc buffer) were thawed and filtered (Millex filter, 0.22 $\mu \mathrm{m}$ ). If need be, buffer exchange was implemented by dialyzing (in Slide-A-Lyzer, 3.500 MWCO, Thermo Scientific) against either "NaCl" or "His" buffer. Dialyzed solutions were filtered again (sterile Minisart filter, $0.22 \mu \mathrm{m}$, Sartorius) into sterilized tubes. Samples were then incubated in thermostated ovens at either $25^{\circ} \mathrm{C}$ or $40^{\circ} \mathrm{C}$ for accelerated ageing. Final concentrations were determined from absorbance (Nanodrop 2000) knowing the extinction 
coefficients of both $\mathrm{mAb}$ and ADC see "Calculation of the molar mass" in Supporting Information, and $\operatorname{Ref}^{7}$.

Ultra-Performance Liquid Chromatography. UPLC measurements were performed on a ACQUITY UPLC H-Class Bio (Waters) using a Waters Acquity UPLC BEH200 column (30 cm long) The mobile phase consisted of $10 \%(\mathrm{v} / \mathrm{v})$ acetonitrile and $90 \%(\mathrm{v} / \mathrm{v})$ of a solution of 100 $\mathrm{mM}$ Tris- $\mathrm{HCl}, 440 \mathrm{mM}$ sodium perchlorate, $\mathrm{pH} 7.9$ that was flushed at $0.3 \mathrm{ml} / \mathrm{min}$ flow rate and $30^{\circ} \mathrm{C}$ for a typical run time of $15 \mathrm{~min}$. Injection volume was $4 \mu \mathrm{L}$ of samples diluted in the mobile phase to reach an injected amount in the range 10-20 $\mu \mathrm{g}$ IgG. The relative surface areas given in Table 1 is the $\%$ areas of peaks normalized by the sum of all peaks accounting detected in the chromatogram by absorbance at $230 \mathrm{~nm}$.

SEC-SAXS separation and measurements. Size-exclusion chromatography coupled to smallangle X-ray scattering (SEC-SAXS) measurements were performed on the BM29 beamline at the European Synchrotron Radiation Facility (Grenoble, France) using two SEC columns (4.6 mm $\times$ $300 \mathrm{~mm}$, TSK Super SW3000, Tosoh Bioscience) mounted in series on a high-performance liquid chromatography (HPLC) device (Shimadzu NexeraXR) coupled to the SAXS flowthrough quartz capillary cell. The mobile phase consisted of $10 \%(\mathrm{v} / \mathrm{v})$ acetonitrile and $90 \%(\mathrm{v} / \mathrm{v})$ of a solution of $100 \mathrm{mM}$ phosphate, $300 \mathrm{mM}$ sodium perchlorate, $\mathrm{pH} 6.2$ that was always flushed at $0.3 \mathrm{ml} / \mathrm{min}$ flow rate and $20^{\circ} \mathrm{C}$. The use of perchlorate and acetonitrile enhanced solubility of aggregates and avoided bias due to adsorption onto the stationary phases. The columns were equilibrated ex situ for $3 \mathrm{~h}$ and then mounted on the X-ray-HPLC line and flushed for $1 \mathrm{~h}$ with the 
mobile phase prior to the first injection. SAXS patterns were recorded using a Pilatus 1M detector. The sample-to-detector distance was set to $2867 \mathrm{~mm}$ and the energy $\mathrm{E}=12.5 \mathrm{keV}$ which provided scattering wavenumbers $q=(4 \pi / \lambda) \cdot \sin (\theta / 2)$ (with the wavelength $\lambda=0.99 \AA$ ) ranging from $3.6 .10^{-3}$ to $0.49 \AA^{-1}$. The SAXS data acquisition was set to 1 frame/s and the injection volume of samples was $100 \mu \mathrm{L}$ unless specified otherwise. SAXS data at the BM29 beamline was processed through a set of on-site dedicated softwares that operated all data reduction, azimuthal integration, curve merging and background correction ${ }^{20}$. Intensity data were converted in absolute scale by comparison with the scattering from pure $\mathrm{H}_{2} \mathrm{O}$. Additional SAXS analysis were carried out with the softwares GNOM ${ }^{21}$ and PRIMUS ${ }^{22}$ such as the calculation of the distance distribution function $\mathrm{p}(\mathrm{r})$ and Guinier fitting. The molecular mass was estimated from a Guinier fit knowing the concentration from the absorbance data (see calculation in Supporting Information) as well as from Porod volume $V_{p}$ as $V_{p} / 1.6$ using PRIMUS ${ }^{22}$. Singular Value Decomposition (SVD) and Evolving Factor Analysis (EFA) were employed to ensure no contamination occurred when extracting species of interest by using the BioXTas Raw software. $^{23}$

Ab initio and rigid body modelling. Three-dimensional (3D) reconstructions of monomers and dimers were carried out by two methods: $a b$ initio and rigid body modeling. For $a b$ initio modelling, we used DAMMIN software ${ }^{24}$ to determine low-resolution envelopes made of densely packed beads. This program minimizes the fit residual between experimental scattering intensities (SAXS patterns) and calculated scattering by a dummy compact structure generated by the DAMMIN simulated annealing procedure. For each reconstruction, twelve independent runs were performed and then averaged upon application of DAMAVER software. ${ }^{25}$ This first output was used as a starting model for twelve subsequent runs on DAMMIN. The final structure 
was thus obtained with a total of two refinements. No symmetry was imposed in any of the reconstructions.

For rigid body modeling, we used the software CORAL ${ }^{26}$ and structures of mAb fragments that are typical of IgG1 (obtained from PDB 1IGT; the Fc and Fab sequences of our mAb and the PDB 1IGT have respectively $100 \%$ and $60 \%$ identity). The monomer model was composed of four domains treated as rigid bodies: two identical Fab domains, one Fe domain, and the hinge region (with disulfur bonds). The Fabs consisted of residues 1-214 (chains A and C) and 1-228 (chains B and D), the hinge of residues 236-243 (chains B and D) and the $\mathrm{Fc}$ of residues 248-474 (chains B and D). To allow in silico explorations of conformations, these rigid bodies were connected by flexible linkers (consisted of dummy residues as described by Petoukhov et al. ${ }^{26}$ to replace the intentionally omitted residues). The output from a run of CORAL was then a physically permitted conformer (no superimposition of residues) that locally minimizes the scattering difference between simulated SAXS pattern and experimental SAXS data. Three independent runs were systematically performed using default parameters and no additional constraints others that those already embedded in the program were added (no particular symmetry and no forced contacts).

The final models were rendered by the program CHIMERA ${ }^{27}$. The program SUPCOMB ${ }^{28}$ was used to superimpose the 3D models from CORAL and DAMMIN. After successful reconstruction, both softwares yielded the goodness of fit (reduced chi-square) as defined as $\chi^{2}=\frac{1}{N-1} \sum_{n=1}^{N}\left(\frac{I_{n}-I_{n}^{f i t}}{\sigma_{n}}\right)^{2}$, where $I_{n}, I_{n}^{f i t}$ and $\sigma_{n}$ represent respectively the experimental scattering intensities, computed intensities from the model and the experimental standard deviations. 
Atomic force microscopy. Atomic force microscopy (AFM) images were obtained in air at room temperature. A freshly cleaved mica surface (potassium aluminosilicate Muscovite Mica from Goodfellow) was first treated with $1 \mathrm{mM}$ spermine solution for 7 minutes and rinsed with an excess of water. After water removal with filter paper (Whatman), a $30 \mu \mathrm{L}$ drop of $1 \mu \mathrm{g} / \mathrm{mL}$ protein solution was deposited on the mica for 1 minute. Finally, the surface was rinsed abundantly with water and dried by blowing air. AFM measurements were performed using a 5100 Atomic Force Microscope (Agilent Technologies-Molecular Imaging) operated in a dynamic tip deflection mode (Acoustic Alternating Current mode, AAC). All AFM experiments were performed using silicon probes (Applied NanoStructures-FORT) in the tapping mode with a spring constant of $3 \mathrm{~N} \mathrm{~m}^{-1}$ and having typical frequency in the range $60-65 \mathrm{kHz}$. AFM images are displayed with a height scale between $-1.2 \mathrm{~nm}$ and $+1.7 \mathrm{~nm}$.

\section{Results}

Analysis of monomer and soluble aggregates.

Monomer and soluble aggregates were generated upon ageing in accelerated storage conditions at $25^{\circ} \mathrm{C}$ or $40^{\circ} \mathrm{C}$. After an incubation for 48 days in different buffer conditions, we characterized the slowly formed oligomers by size-exclusion chromatography using a carrier fluid that minimized IgG binding on the stationary phase (see material \& methods section). We used UPLC and a small injected amount of samples (see method section) to achieved higher resolution of the peaks as compared to HPLC (Fig. 1A). A single species corresponding to the monomer was detected at a significant peak amplitude from fresh, non-aged ADC (or mAb) solutions. Detection of additional peaks at time shorter than this monomer peak thus indicates the 
presence of non-labile oligomers (i.e. that were not equilibrium assemblies with monomers but were formed during ageing). The relative amounts of monomer and oligomers were calculated as the $\%$ area of absorbance peaks at $230 \mathrm{~nm}$ in chromatograms and are shown in Table 1 . The maximum amount of dimer (9.4 and $9.8 \%$ area resp.) were formed in 48-day-old ADC solutions kept at $40^{\circ} \mathrm{C}$ in respectively $20 \mathrm{mM}$ Histidine buffer $\mathrm{pH} 6.0$ (His buffer) or in $5 \mathrm{mM}$ sodium acetate-acetic acid pH 5.0 (NaAc buffer). These two samples also contained the maximum \% of larger oligomers ( $12.3 \%$ and $6.6 \%$ resp.). As compared to $\mathrm{mAb}$ solutions in the same incubation conditions $(<4.8 \%$ dimer and $<0.2 \%$ larger oligomers $)$, ADC was more prone to form clusters and HMWs. Ageing of $\mathrm{ADC}$ or $\mathrm{mAb}$ at $25^{\circ} \mathrm{C}$ for 48 days, either in the His buffers or in $\mathrm{NaCl}$ buffer (136 mM NaCl with $20 \mathrm{mM}$ sodium acetate-acetic acid $\mathrm{pH} 5.0)$ yielded similarly higher level of dimer and oligomers in ADC solutions as compared to $\mathrm{mAb}$, but in markedly lower amount as compared to incubation at $40^{\circ} \mathrm{C}$ (typically $<3.3 \%$ dimer, and $<1.2 \%$ large oligomers).

To prepare fractions of purified oligomers in sufficient amount for SAXS scattering, the aged samples were separated by HPLC using an adapted, though similar, elution buffer. The HPLC profiles were typical of patterns commonly found in literature for aged mAbs and ADCs. The predominant peak (here centered at time $1100 \mathrm{~s}$ on UV absorbance trace) was attributed to the monomer, based on the calculated molar mass of $145 \mathrm{kDa}$ from Guinier analysis and $164 \mathrm{kDa}$ from Porod volume (Fig. 1, Table 2, and Fig S1 in supporting). At shorter elution times, two minor peaks could be detected on the UV absorbance trace: one at $950 \mathrm{~s}$ assigned to dimers (molar masses 275-283 $\mathrm{kDa}$ from Guinier in Table 2) and a second one before ca. $900 \mathrm{~s}$ corresponding to ill-defined apparent molar masses that suggested a slow drift and mixtures of 
trimers up to large oligomers (10-12 monomer units). One additional minor peak at longer elution times $(>1300 \mathrm{~s})$ was attributed to protein fragments and not analyzed further.

To maximize the scattered intensity in SAXS and enable the record of reliable signals (well above noise) from the minor fractions of dimers and HMWs, a large injection volume (up to 100 $\mu \mathrm{L}$ ) was needed which decreases the chromatographic resolution. As a consequence, the peaks of monomer, dimers and HMWs species were partly overlapped in these conditions (Fig S1 in Supporting Information). Automated estimates of the radius of gyration $R_{g}$ could be obtained all along the chromatogram trace, by using the dedicated data processing pipeline on BM29 beamline. ${ }^{20}$ As shown in Figure 1, the left-hand side of monomer peak (1070-1100 s) can be fully separated from dimers (fractions between 950-1000 s) as judged qualitatively from the constant radius and molecular weight in each window (Fig. 1 and Table. 2). In contrast, a drift of $R_{g}$ was obtained along the peak of HMWs possibly showing in some samples (as in Fig. 1) a contamination between HMWs and the earliest fraction of dimers (which was due to the loss of resolution at high injection volume). When this contamination occurred, SAXS profiles of dimers were calculated from data recorded in the 950-1000 s time window, i.e. in the right-hand part of the peak. Superimposition of instantaneous profiles (collected every 1s during elution) shows in Fig. 1C the absence of drift in data recorded in the monomer and dimer fractions. Singular Value Decomposition (SVD) and Evolving Factor Analysis (EFA) were applied on these data to ensure no contamination occurred within the corresponding timeframe (Fig. S2, S3, S4 and S5 in Supporting Information). The spectra of larger oligomers could not be isolated: a gradual variation of SAXS profiles was recorded in the 810-900 s window of HMWs confirming the elution of gradually larger oligomers, that are likely poorly resolved in this time window. This is also shown in the evolution of radius of gyration and molecular masses (Fig. S1). 
Because of poor resolution between HMWs, their apparent mass along the elution time (estimated by Guinier fitting) gradually varied from the molecular weight (MW) of trimers up to that of 12-mers (with a $R_{g}$ up to $\sim 15 \mathrm{~nm}$ when aged in the His buffer).

Another feature in the SEC-SAXS separation is the small elution peak detected in the 710-750 s window by SAXS in all buffers (Figures 1A and S1) but not by UV-Vis absorbance. The SAXS absolute intensity is proportional to the molar concentration times the square of the molar mass. This elution peak was thus attributed to the detection of a small amount of very large aggregates. This peak was detected in all buffers and even when no samples were injected through the column (blank injection of buffer), which suggests that the HPLC columns were releasing aggregates. To assess the presence of large aggregates in aged samples (and avoid artefacts due to desorption from the HPLC columns), we fractionated a representative solution of ADC through a single-use freshly prepared gel (Sephacryl@ S-500HR packed in a $100 \times 10 \mathrm{~mm}$ glass column, flushed at $0.1 \mathrm{psi}$, collected fractions of $0.2 \mathrm{~mL}$ ) with the same elution buffer as for HPLC. Though with a much lower resolution than HPLC, the fractions collected from this gel corresponded to i) elution volumes of aggregates that were excluded from the gel, ii) intermediate species that migrated through the gel, iii) a final fraction with a predominance of monomers. We observed that the SAXS patterns of the first eluted volume after the columns' dead volume (i.e. $0.8 \mathrm{~mL}$ in Fig. S7 in supporting) can be matched with the SAXS patterns of species that were eluted first in the HPLC column at $710 \mathrm{~s}$. Further analysis on their form factors show that they could be modeled as mass fractals in order to primarily estimate their fractal dimension Df. Values of Df 2.6-2.7 (see Fig. S8 in Supporting Information) lead to the conclusion that these largest aggregates were quite compact. 
Structures of the monomers before and after ageing.

Averaged SAXS profiles normalized by the forward scattering intensity are presented in Figure 2A for the SEC fraction of ADC monomer collected from samples incubated for 48 days in the different buffers. All these curves were undistinguishable within experimental error. A profile collected from a 2-day-old ADC sample which was injected in the SAXS capillary in static mode (no HPLC, ADC freshly prepared in NaAc buffer) is also shown in supporting data for comparison (Fig. S9). We note a subtle difference at $\mathrm{q} \sim 1 \mathrm{~nm}^{-1}$ between fresh and aged monomers which may betray slight evolutions at the length scale of individual residues, such as minor chemical degradation. Further, to compare the monomer of ADC with $\mathrm{mAb}$, an aged sample of mAb formulated in NaAc buffer was eluted using the same column in the same conditions. The SAXS profile of mAb also slightly differed from the one of ADC which becomes clear in the Kratky representation (Fig. 2B). A possible origin of this difference is the contribution to scattering of drug conjugates. The asymptotic decay in the high $q$ range is consistent with a well-defined multidomain protein, allowing for the calculation of the pair distance distribution function p(r) (Fig. 2C). To achieve the latter calculation, we implemented the software GNOM ${ }^{21}$ validated for common globular proteins. The resulting 15-16 nm maximal extension of ADC monomer is compatible with known structures of native IgG. Using $\mathrm{p}(\mathrm{r})$, it was possible to construct low-resolution structures. On one hand, we implemented ab initio reconstructions using the software package DAMMIN ${ }^{24}$ (see method section). After multiple independent runs and two refinements, we ended up with a structure made of packed beads that converged to a typical T-shape antibody-like envelope. On the other hand, an alternative structure was converged by random exploration of the relative positions of two Fab and one Fc 
domains treated as rigid bodies, connected by flexible linkers (using CORAL package ${ }^{26}$, see method section). Both reconstructions yielded excellent fits as shown in the $\log (I)$ vs $q$ representation (Fig 2D). It is remarkable that the two putative structures could be matched together by ad hoc spatial rotations (Figure 2E, 2F). In particular, within a reasonable estimate of $\sim$ nm resolution, the distal tips of the three domains appeared to occupy the same positions and to be in similar co planar configuration in the two reconstitutions. Because of the flexibility of $\operatorname{IgG}$, the SAXS spectrum of monomer is certainly an average of the scattering from several conformers in dynamic equilibrium. Convergence of 3D reconstruction primarily suggests that the present envelops provide a fair visualization of the average distribution of mass among the predominant conformations present in solution. With no need for more refined analysis, it can be concluded that after ageing in any of the three buffers, the (average) low-resolution structural features of ADC monomer did not significantly differ from the one observed in fresh samples.

Structural features of soluble aggregates (dimers).

A similar data treatment was applied on the SAXS data recorded in the elution time window corresponding to dimers. In Figure 3A, the highlighted points within the peak of dimers indicate the time window where $R_{g}$ and apparent Mw were constant. In ADC samples containing larger $\%$ of HMWs this range was limited to the right-hand side of the peak due to contamination by larger oligomers in the left-hand side. Typical features shown in Table 2 pointed to a remarkable similarity between $\mathrm{ADC}$ dimers formed in the different buffers $\left(R_{g} \sim 7.5 \mathrm{~nm}, \mathrm{D}_{\max } \sim\right.$ $27 \mathrm{~nm}$ ). The relevant time window could be extended to the left-side of the peak in mAb samples ( $\sim 930-1000$ s), presumably because of a lower content of large oligomers. The instantaneous 
SAXS profiles were further averaged for noise reduction (Figure 3B and S6 in supporting information). With a radius of gyration $R_{g}$ of $9.6^{+/}-0.6 \mathrm{~nm}$ the dimers of mAb appeared more elongated compared to ADC ones. A difference between the ADC and mAb dimers can already be seen in these profiles and from $R_{g}$, and it is enhanced in the dimensionless Kratky plot (Fig. 3C). The first maximum in Figure $3 \mathrm{C}$ for $\mathrm{ADC}$ is reached at $q \cdot R_{g}=2$ which is slightly shifted from the coordinate $(1.7,1.1)$ expected for well-defined globular proteins. ${ }^{29,30}$ Of note, the position of this maximum in the dimensionless Kratky plot of ADC monomer (Fig 2B) does not markedly differ from the one of dimer, suggesting that IgG flexibility per se is sufficient to achieve this slight shift. The profiles of ADC dimers obtained from samples aged in the three different incubation buffers could be superimposed (Fig. 3D), suggesting that similar structures were reached irrespective of ageing conditions. In contrast, for the dimers of $\mathrm{mAb}$, the first maximum in the dimensionless Kratky plot is way above 1.1 at $q \cdot R_{g}=1.7$. This suggests a significantly less compact structure as compared to ADC dimers, and/or significantly different structural diversity in the population of mAb dimers.

$A b$ initio reconstructions and rigid body (with flexible linkers) modelling were applied to fit the SAXS patterns of dimers in NaAc buffer. Representative structures are shown in Fig. 3E, with the corresponding theoretical SAXS that fitted experimental data up to $\mathrm{q}=1.5 \mathrm{~nm}^{-1}$ (Fig. 3B). The matching between the two models was not as good as for the monomers (in particular in the case of mAbs dimers, which is not surprising for an ill-defined population of flexible objects) as seen from the deviation above $\mathrm{q}=1.5 \mathrm{~nm}^{-1}$ in the Kratky plot (Fig. 3C). To visualize low-resolution models of the spatial distribution of scatterers in the dimers, we used CORAL and let the algorithm find the best arrangements between the two monomers (no forced contacts were imposed at any time). A strong constraint is thus introduced by rigid-body (rather than dummy 
atoms) modelling, making the CORAL method more suitable to generate physically plausible, though putative structures. Here, as for the monomer modeling, the 3D reconstructions provide visualizations of the SAXS-averaged structural information embedded in the data and enables to conveniently compare variations due to different ageing conditions. For ADC dimers, all CORAL-converged averaged structures (Fig. S10) contained a dense central body made of 3-4 ( $\mathrm{Fc}$ and/or Fab) domains and involving several contacts between the monomers. Among the contacts points, some were located on the side of the central hinge. This central body was flanked by "peripheral", contact-free domains (two of them typically placed along a diametral axis of the structure). Computer-generated solutions displayed tighter contacts for ADC as compared to mAb dimers that typically showed one contact area located in the "outer part" of domains (i.e. the half part of domains away from the hinge, Fig S11). In this line, Plath et al. ${ }^{31}$ applied SAXS studies (combined with TEM and biochemical characterizations) on IgG mAb dimers formed upon either thermal stress or ageing at room temperature. Different SEC-purified fractions were revealed to contain either elongated dumbbell-like objects, or compact dimers shedding light on possible association-prone domains. Although the study by Plath et al. was based on a peculiar case where two dimers could be separated by SEC (presence of two peaks of dimers in chromatograms, which was not observed in our samples), the elongated dumbbell-like objects may share some common feature with the elongated $\mathrm{mAb}$ dimers that were formed in our samples.

Due to the denser central region and innermost contacts simulated in ADC dimers, it is not surprising that orientations and/or angles between domains were in addition markedly affected, thus diverging from the native-like conformation of the ADC monomer. An evidence for distortion is that all tentative fits based on random exploration of relative orientations of two 
rigid-like monomers (i.e. monomers preserving the envelope as determined in Fig. 2 and treated as a rigid bodies) did not match with the experimental profiles (Fig S12), suggesting that the introduction of inter-domain flexibility in the hinge of each ADC monomers was key for convergence. It is concluded that despite the low resolution and averaging of the structural information (in particular average among a likely disperse population of conformers), the differences in extension and compactness between $\mathrm{mAb}$ and $\mathrm{ADC}$ dimers can be interpreted by strong structural constrains on the relative positions of domains: the higher Dmax and Rg of $\mathrm{mAb}$ dimers can result from outermost inter-mAb contact between $2 \mathrm{Fab}$ or $\mathrm{Fc}$ domain, whereas 3-4 Fab and/or Fc domains in tight contact shall be in the center of the ADC dimers.

Structural variability assessment by AFM.

To complement structural assessment from SAXS data, we carried out Atomic Force Microscopy (AFM) imaging. This technique has been recently used to characterize conformational diversity of adsorbed mAb monomers ${ }^{32,33}$. We immobilized the antibodies by spontaneous adsorption from water solutions on mica sheets that were coated with spermine (bath application of a fraction collected from the SEC elution column, see method section). Dilution of the deposited protein solution was adjusted to balance the random overlap between proteins with the highest possible density of adsorption. The dried mica plates were scanned in air, in tapping mode (Figure 4A). The population of objects that adsorbed flat onto the mica (typical averages over about 200 isolated objects gave heights as follows: mAb monomer $1.38 \pm$ $0.27 \mathrm{~nm}, \mathrm{mAb}$ dimer $1.54 \pm 0.42 \mathrm{~nm}, \mathrm{ADC}$ dimer $1.53 \pm 0.36 \mathrm{~nm}$ ) was analyzed by Image $\mathrm{J}$ to 
record their largest diameters (maximum Feret's diameter of each object) and their aspect ratios. Whether the data include some contribution of variability in the adsorption/orientation of IgG on the surface, or fairly represent the distribution of sizes in solution cannot be determined here. We assumed however that any variability due to surface immobilization should have similar impacts on species having similar structural features. The collected apparent lengths are used here to compare qualitatively the degree of size dispersity and/or compactness. The corresponding size distributions curves shown in Figure $4 \mathrm{~B}$ confirmed that $\mathrm{mAb}$ dimers have on average a larger diameter than $\mathrm{ADC}$ ones and that the AFM resolution was sufficient to distinguish monomers from dimers. The control experiment with mAb monomer indicated in addition that a typical half-width of $5 \mathrm{~nm}$ is representative of a well-defined monodisperse protein. In this line, the monomer size distribution appeared only slightly sharper than ADC dimer ones. Dot plot of diameters $v s$ aspect ratios in Figure 4C helped to further estimate conformational variability. In this representation, the population appeared as a broad cloud around the average size parameters. The broadest and most elongated cloud was displayed by mAb dimers whose aspect ratio varies from 1 up to 2.4, suggesting coexistence of diverse, more or less elongated, conformations. In contrast, a denser cloud of dots characterized the monodisperse monomers, with apparent aspect ratios lower than 1.6 (variations may be due to diverse orientations of the adsorbed molecules). We noticed a few dots away from the predominant population, that were likely due to unfiltered overlaps between two monomers as they show typical diameter of dimers (see method section). These few events did not significantly contribute to the main features of the whole population. Dot plot for ADC dimers was significantly more dense than the one of $\mathrm{mAb}$ dimers. Most objects had an aspect ratio below 1.8, and most typically below 1.6 suggesting a rather compact configuration whose diversity was not markedly higher than the one observed on monomers. 
Finally, AFM images suggest that the HPLC fraction of ADC dimer contained a population of rather compact objects, whereas the fraction of $\mathrm{mAb}$ dimer displayed much more heterogeneous conformations.

\section{Discussion}

A first important remark is the preservation in the monomer fraction of the native-like shape, for up to 48 days at $25^{\circ} \mathrm{C}$ or $40^{\circ} \mathrm{C}$. Irrespective of the condition of ageing and the $\mathrm{pH}$ buffering system, the SAXS profiles in the HPLC fraction of monomers were essentially identical and resemble the monomer freshly thawed. It has been established, in the case of $\mathrm{mAb}$ monomers, that SAXS is a sensitive technique to detect transition of conformations for instance upon addition of osmolytes or variations of $\mathrm{pH}^{15,16,34}$. Changes in the envelope of $\mathrm{mAb}$ may occur without denaturation as reversible equilibria between "Y" and "T" shapes and can be characterized by variation in SAXS ${ }^{15,35}$. These few reports in literature suggest that in usual buffers, $\mathrm{mAb}$ monomers can keep a $\mathrm{pH}$-independent and concentration-independent equilibrium conformations (as characterized by an average structural information embedded in the SAXS), a conclusion that we also proposed in the case of fresh samples of ADC (in the experimental window of $\mathrm{pH} 5-6$ and $<30 \mathrm{~g} / \mathrm{L}$ ADC) ${ }^{7}$. Here, however the goal was to study whether irreversible change may affect the proteins after a 48-day long storage. The predominance of the same native-like, average envelope in samples stressed for days at either $25^{\circ} \mathrm{C}$ or $40^{\circ} \mathrm{C}$ contributes to validate a common assumption made in the nucleation-growth theories of protein aggregation: i.e. absence of significant amount of conformational change and rare events of destabilization/fast aggregation amid a large pool of essentially native-like monomers. 
The structural compactness of ADC dimers that were formed during ageing contrasts with the more elongated, likely more polydisperse dimers formed by the parent $\mathrm{mAb}$ in the reference buffer. The relative compactness of ADC dimers as opposed to mAb dimers was also suggested by AFM. In addition, despite different experimental conditions that led to obvious sample-to-sample variations of kinetics (by a factor of 5) of monomer loss under diverse $\mathrm{pH}$ buffer systems, and despite markedly different final HMWs / dimer molar ratios (Table 1), it is remarkable that a single SAXS pattern was obtained for the ADC dimers. The convergence of $a b$ initio envelope reconstructions with structures obtained by spatial adjustment of rigid Fab and Fc-like domains enable to interpret the difference in Rg and Dmax between mAb and ADC dimers. Of note, good superimposition of these two simple models at nm resolution does not preclude that structural variability exist (for instance, variability in interdomain arrangement, and flexibility is clearly suggested by the shape of dimensionless Kratky plots). The dispersities possibly present in solution are here "averaged" in the models, providing a fingerprint of a global redistribution of protein domains. The compactness of the model structure of ADC dimers is unlikely to be reached without important constraint on interdomain positions. The convergent structure in the dimer corresponded to a significant distortion compared to the native-like monomer shape, which is consistent with the need to gather a significant mass toward the center of the dimer. Namely, in the putative low-resolution structures, domains from one monomer form tight contacts with the second monomer and can reach the central areas close to the hinge of the second monomer (Fab or Fc cannot be distinguished at this resolution). This result together with the more extended shape of the dimers in mAb solution observed by AFM provide evidence for a significant contribution of conjugation of the $\mathrm{mAb}$ in the aggregation of ADC. It is in particular not possible to explain such structural differences by a simple slowing down of 
similar aggregation pathways for both $\mathrm{mAb}$ and ADC. Strong binding between innermost areas in $\mathrm{ADC}$ dimers are required to reach the observed compaction as compared to mAb. A possible origin of tighter ADC dimer is that covalent conjugations on lysine side groups introduced specific binding-prone areas, including in the innermost regions of the monomer near the hinge.

\section{Conclusions.}

For the first time to our knowledge, structural features of irreversible dimer and oligomers formed in solutions of aggregation-prone ADC were characterized by HPLC coupled to SAXS. After 48-day long incubation at $25^{\circ} \mathrm{C}$ or $40{ }^{\circ} \mathrm{C}$, the predominant protein fraction was identified as monomer that displayed a preserved native-like conformation. Results on dimer characterizations by SEC-SAXS and AFM point to a significantly more compact ADC dimer, and formation of tighter inter-monomer contacts in $\mathrm{ADC}$, as compared to the mAb dimers. The unexpected conclusions of this study is that 1) the same profile of SAXS scattering by dimers is obtained from ADC samples aged in various buffer conditions, despite markedly different $\%$ of HMWs formed in the various samples (see also ${ }^{7}$ for the effect of buffer on the aggregationprone population of $\mathrm{ADC}$ ) and 2) obvious differences between the average structural features of $\mathrm{ADC}$ dimers and $\mathrm{mAb}$ dimers. Based on these results, it becomes possible to argue for a strong contribution in ADC dimers of the chemically modified sites at the conjugation point. Compared to $\mathrm{mAb}$, the conjugation presumably introduces additional possibilities of inter-domain tight binding which is shown here to drive inter-digitations of Fab and Fe domains. The fact that conjugation significantly affected the structure of the dimer, whereas variation of buffer condition did not, suggests that focusing on the parent mAb stability may overlook important 
and specific behavior of $\mathrm{ADC}$ (for instance, formulation studies seeking to optimize mAb by enhancing long-range repulsions do not take into account the possible role of an aggregationprone subpopulation of ADC imparted with specific interaction and/or dimerization patterns). This raises the question of identifying the impact of particular conjugation spots, namely regions on the protein whose modification may have significant impacts on inter-protein binding. During early candidate selection and in particular to screen the role of conjugation procedures, SAXS provides a mean to identify ADCs showing distinctive oligomerization patterns as compared to $\mathrm{mAb}$, without the need for cumbersome analysis of each single conformation present in the solutions.

\section{FIGURES}
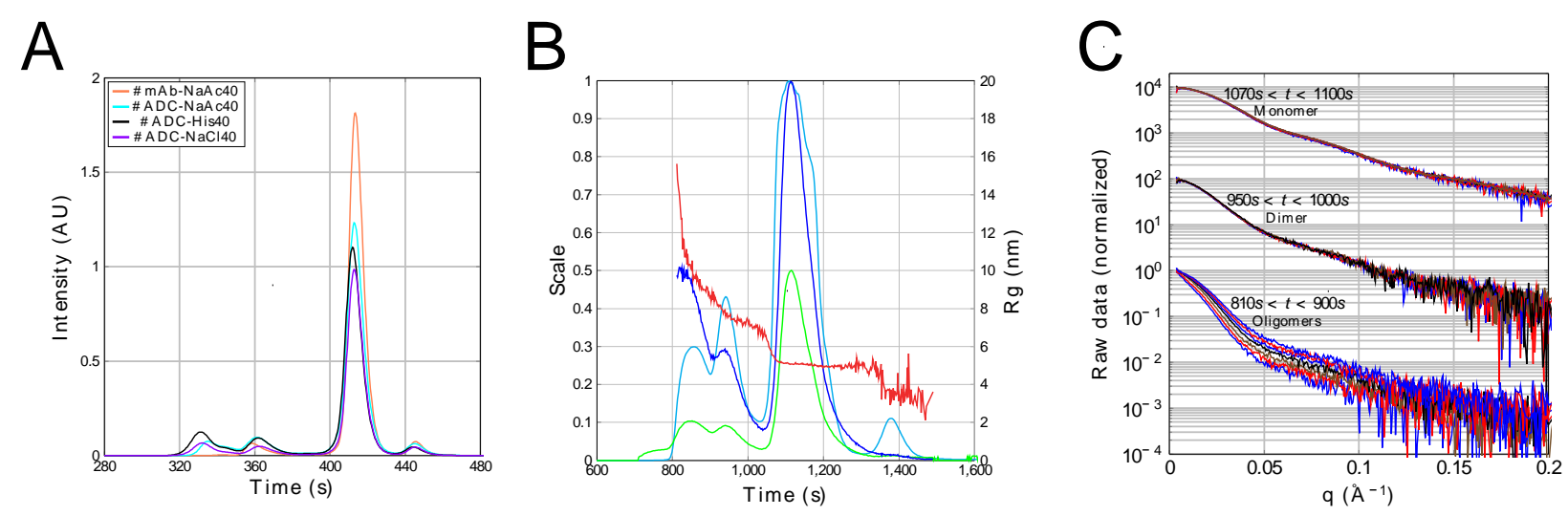

Figure 1. (A) UPLC chromatogram of $\mathrm{mAb}$ and $\mathrm{ADC}$ incubated for 48 days at $40^{\circ} \mathrm{C}$ in different buffers. (B) SEC-SAXS chromatogram of ADC incubated for 48 days at $40^{\circ} \mathrm{C}$ in $\mathrm{NaAc}$ buffer (sample \#ADC-NaAc40) displaying the total subtracted scattering intensity (green line, sum of buffer-subtracted scattered intensities over all experimental angles), the forward scattering I0 
(blue, from Guinier fit), absorbance at $280 \mathrm{~nm}$ (cyan) scaled in arbitrary units (left y-axis); scaling factors are respectively 71.745 (into cm-1), 0.527 (into cm-1) and 2.755 (arbitrary units); the radius of gyration (Rg from Guinier fit, red plot) is expressed in nm (right y-axis). (C) SAXS patterns collected at different elution times obtained from (B). Data were normalized by the forward scattering intensity and vertically shifted for clarity.
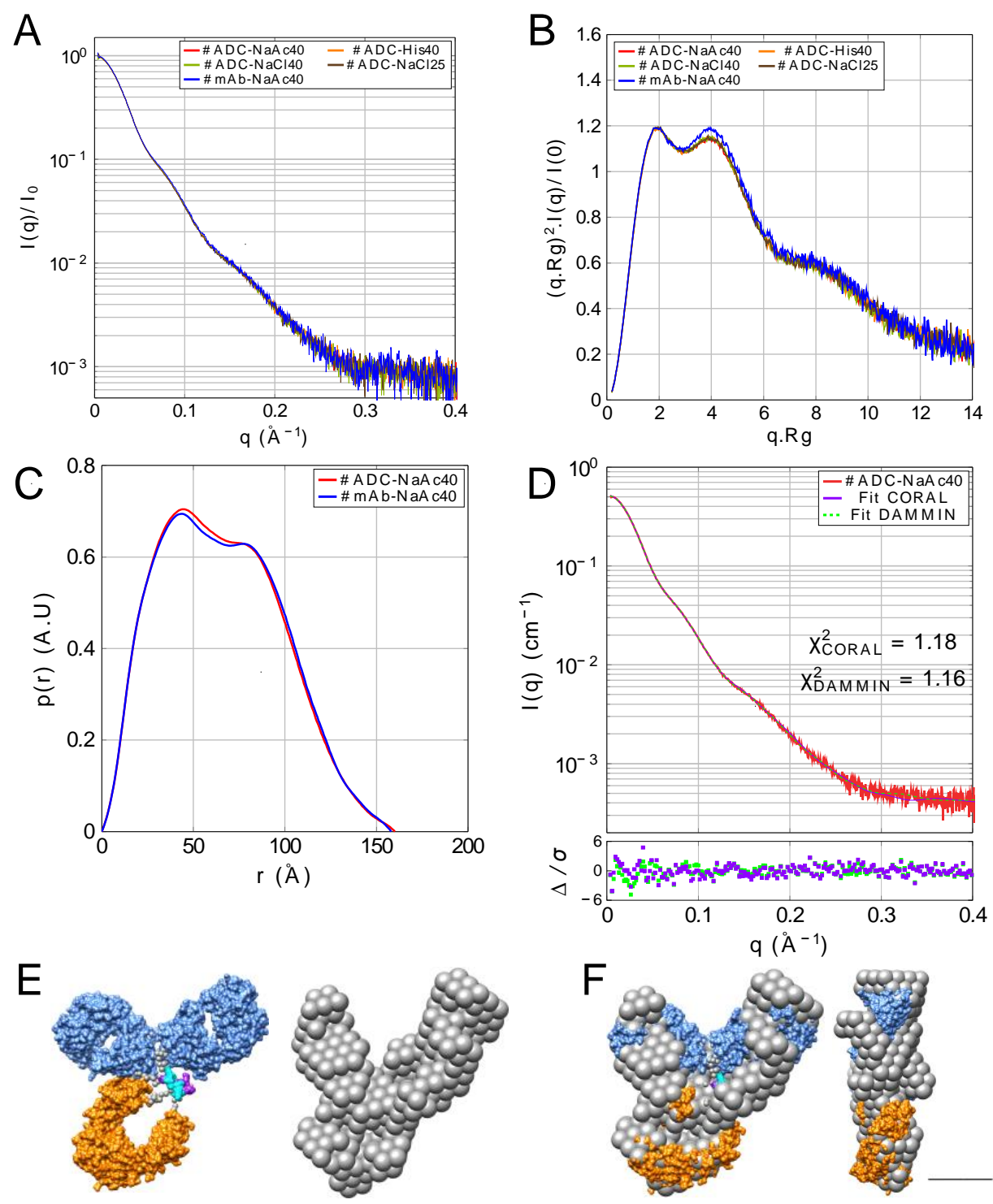
Figure 2. Monomers of $A D C$ and $m A b$ in different buffers. (A) Apparent form factors $I(q) / I_{0}$ of monomers. (B) Corresponding dimensionless Kratky plots; q. $\mathrm{gg}=4$ corresponds to $\mathrm{q}=7.710^{-2} \AA^{-}$ 1. (C) Particle distance distribution function $\mathrm{p}(\mathrm{r})$ for $\mathrm{mAb}$ and ADC monomer in NaAc40 buffer. (D) Fitted plots with continuous shaded error region obtained from CORAL (rigid body) and DAMMIN (ab initio). The bottom inset plot is the error-weighted residual difference plot $\Delta / \sigma$ $=\left[\mathrm{I}_{\exp }(\mathrm{q})-\mathrm{I}_{\mathrm{fit}}(\mathrm{q})\right] / \sigma$. (E) Optimized 3D structures of ADC monomer obtained by ab initio 3D shape reconstruction (right) using DAMMIN, or rigid body reconstruction from CORAL (left) with Fab domains in blue color, Fc in orange, a body in the central hinge in cyan and purple, and flexible linkers are represented by small grey beads (see method section). (F) Superimposition of the structures in (E), as obtained from SUPCOMB (front and side view). Scale bar is $5 \mathrm{~nm}$. 

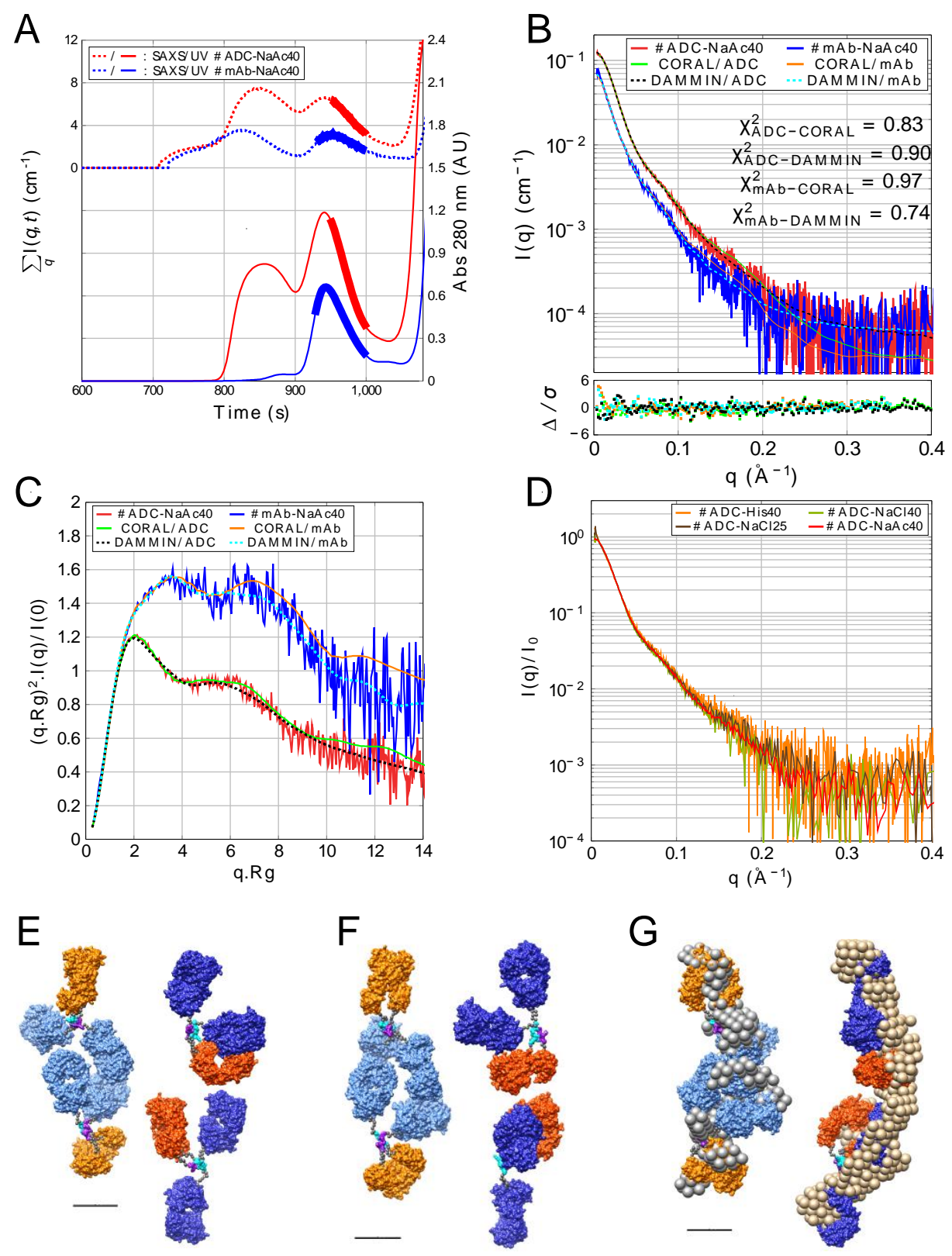

Figure 3. Dimers of ADC and mAb. (A) SEC-SAXS chromatograms (sum of buffer-subtracted intensities over all experimental angles) and UV chromatograms showing the peaks of dimers eluted between $910 \mathrm{~s}$ and $1000 \mathrm{~s}$ and larger oligomers between $700 \mathrm{~s}$ and $900 \mathrm{~s}$. (B) SAXS patterns of the dimers in the regions highlighted by thick solid lines in (A) and fits obtained from CORAL and DAMMIN. Plots are in a continuous shaded representation of error bars. Bottom inset is the error-weighted difference plot. (C) Corresponding dimensionless Kratky. (D) 
Superimposed form factors of the dimers in various ageing buffers. (E-G) Different views of representative 3D reconstructions obtained by CORAL (blue and orange domains) and DAMMIN (grey and gold yellow balls). Dimers of ADCs are shown with a lighter color than dimers of mAbs. Scale bars are $5 \mathrm{~nm}$.
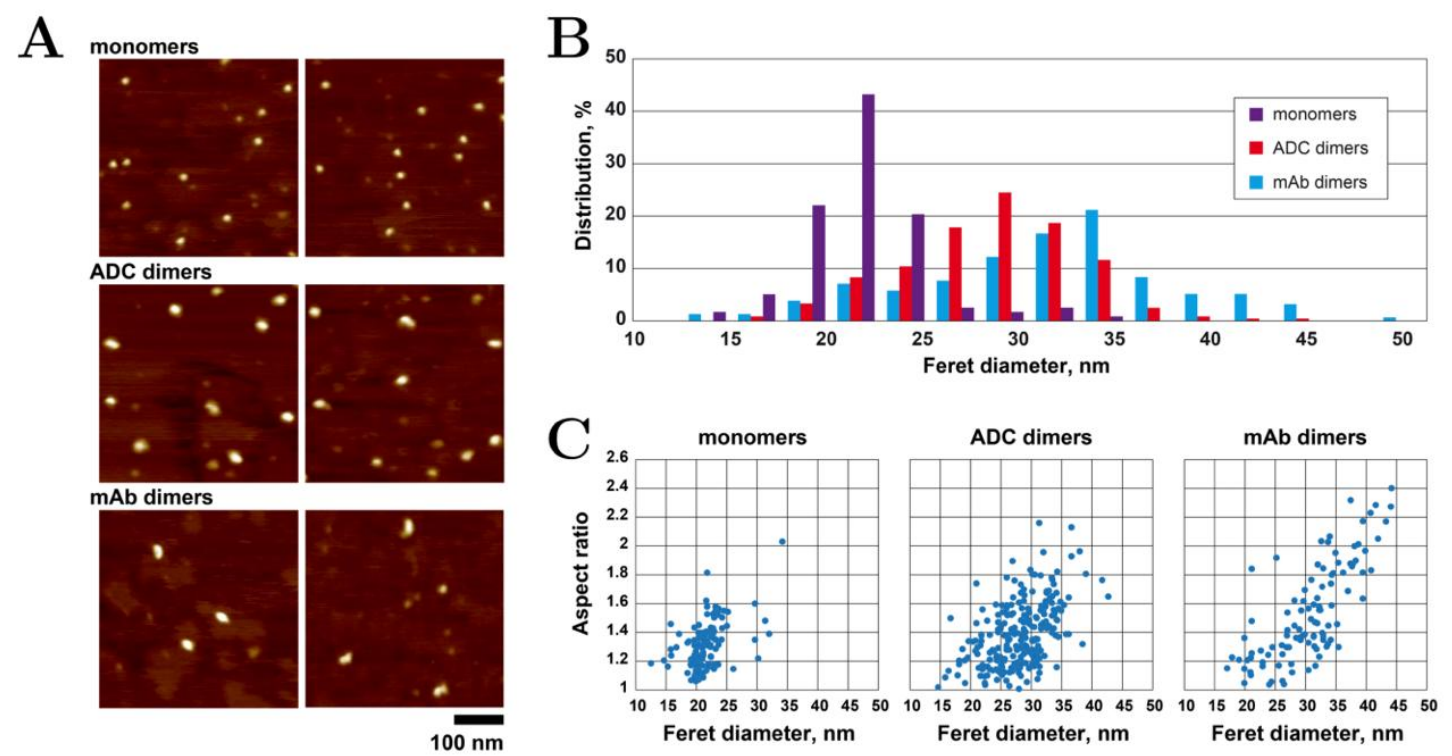

Figure 4. AFM analysis of $\mathrm{ADC}$ and $\mathrm{mAb}$ dimers and $\mathrm{mAb}$ monomers fractionated by HPLC separation from antibodies incubated for 48 days in NaAc buffer $40^{\circ} \mathrm{C}$. (A) Representative images of proteins adsorbed onto spermine-coated mica plates (tapping mode). (B) Number weighted distribution of the larger (Feret) diameter of detected events (over ca. 200 sampled objects). (C) Corresponding dot plot distribution of aspect ratio and maximum Feret diameters of the objects. 
TABLES.

Table 1. Characteristic compositions of representative samples of ADC or mAb after ageing in different buffers

\begin{tabular}{|c|c|c|c|c|c|}
\hline Sample name & $\begin{array}{l}\text { \#mAb- } \\
\mathrm{NaAc40}\end{array}$ & $\begin{array}{l}\text { \#ADC- } \\
\text { NaAc40 }\end{array}$ & $\begin{array}{l}\text { \#ADC- } \\
\text { His40 }\end{array}$ & \#ADC-NaCl40 & \#ADC-NaCl25 \\
\hline Ageing buffer & $\begin{array}{c}5 \mathrm{mM} \mathrm{NaAc} \\
\mathrm{pH} 5.0\end{array}$ & $\begin{array}{c}5 \mathrm{mM} \mathrm{NaAc} \\
\mathrm{pH} 5.0\end{array}$ & $\begin{array}{c}20 \mathrm{mM} \text { His } \\
\mathrm{pH} 6.0\end{array}$ & $\begin{array}{c}20 \mathrm{mM} \mathrm{NaAc} \\
136 \mathrm{mM} \mathrm{NaCl} \\
\mathrm{pH} 5.0\end{array}$ & $\begin{array}{c}20 \mathrm{mM} \mathrm{NaAc} \\
136 \mathrm{mM} \mathrm{NaCl} \\
\mathrm{pH} 5.0\end{array}$ \\
\hline Concentration $(\mathrm{g} / \mathrm{L})$ & 30.5 & 29.5 & 23.1 & 16.5 & 23.1 \\
\hline Ageing temperature & $40^{\circ} \mathrm{C}$ & $40^{\circ} \mathrm{C}$ & $40^{\circ} \mathrm{C}$ & $40^{\circ} \mathrm{C}$ & $25^{\circ} \mathrm{C}$ \\
\hline $\begin{array}{l}\text { Monomer }(\% \text { Area }) \\
\mathrm{t}=0 \text { day } / \mathrm{t}=48 \text { days }\end{array}$ & $98.1 / 89.5$ & $97.7 / 76.8$ & $97.4 / 73.0$ & $98.0 / 79.8$ & $98.0 / 93.9$ \\
\hline $\begin{array}{l}\text { LMW }(\% \text { Area }) \\
\mathrm{t}=0 \text { day } / \mathrm{t}=48 \text { days }\end{array}$ & $1.0 / 5.5$ & $1.2 / 6.8$ & $1.2 / 5.3$ & 1.4 / 6.3 & $1.4 / 1.6$ \\
\hline $\begin{array}{l}\text { HMW1 Dimer }(\% \text { Area }) \\
\mathrm{t}=0 \text { day } / \mathrm{t}=48 \text { days }\end{array}$ & $0.9 / 4.8$ & $1.0 / 9.8$ & $1.3 / 9.4$ & $0.6 / 6.3$ & $0.6 / 3.3$ \\
\hline $\begin{array}{l}\text { HMW2 Oligomers }(\% \\
\text { Area) } \mathrm{t}=0 \text { day } / \mathrm{t}=48 \text { days }\end{array}$ & $0.0 / 0.2$ & $0.1 / 6.6$ & $0.1 / 12.3$ & $0.0 / 7.6$ & $0.0 / 1.2$ \\
\hline
\end{tabular}

Line quoted "concentration" indicates the initial antibody concentration as determined by UV absorbance (see text) before storage at either $25^{\circ} \mathrm{C}$ or $40^{\circ} \mathrm{C}$. The $\%$ of high molecular weight species (HMW) and low molecular weight ones (LMW, i.e. degradation fragments) were determined by SEC-UPLC and UV absorbance detection. 
Table 2. Structural parameters of mAb and ADC species present in the "monomer" and HMW1 peaks as determined from SAXS patterns

\begin{tabular}{|c|c|c|c|c|c|c|c|c|c|c|}
\hline & \multirow{2}{*}{$\begin{array}{c}\text { Monomer } \\
\text { mAb } \\
\text { NaAc40 }\end{array}$} & \multicolumn{4}{|c|}{ Monomers ADC } & \multirow{2}{*}{$\begin{array}{c}\text { Dimer } \\
\mathrm{mAb} \\
\mathrm{NaAc} 40\end{array}$} & \multicolumn{4}{|c|}{ Dimers ADC } \\
\hline & & $\mathrm{NaAc} 40$ & His40 & $\mathrm{NaCl} 40$ & $\mathrm{NaCl} 25$ & & $\mathrm{NaAc} 40$ & His40 & $\mathrm{NaCl} 140$ & $\mathrm{NaCl} 25$ \\
\hline \multicolumn{11}{|l|}{ Guinier analysis } \\
\hline$R_{g}(\AA)$ & $51.5 \pm 0.4$ & $50.9 \pm 0.2$ & $51.3 \pm 0.5$ & $50.7 \pm 0.2$ & $50.8 \pm 0.4$ & $95.7 \pm 5.8$ & $74.4 \pm 1.1$ & $81.38 \pm 7.0$ & $74.4 \pm 3.9$ & $73.7 \pm 3.1$ \\
\hline $\begin{array}{c}M \text { in } \mathrm{kDa}^{\mathrm{a}} \\
\text { (ratio to expected) }\end{array}$ & $148^{\mathbf{b}}(1.0)$ & $145^{\mathbf{b}}(1.0)$ & - & - & - & $310(1.1)$ & $282(0.9)$ & $275(0.9)$ & 283(0.9) & $277(0.9)$ \\
\hline \multicolumn{11}{|l|}{$\mathrm{P}(\mathrm{r})$ analysis } \\
\hline$R_{g}(\AA)$ & $51.4 \pm 0.1$ & $51.1 \pm 0.1$ & $51.3 \pm 0.1$ & $51.0 \pm 0.1$ & $51.4 \pm 0.1$ & $92.7 \pm 1.1$ & $77.0 \pm 0.5$ & $79.3 \pm 1.4$ & $76.4 \pm 1.2$ & $76.6 \pm 1.6$ \\
\hline$d_{\max }(\AA)$ & 158 & 157 & 158 & 157 & 156 & 291 & 272 & 273 & 272 & 267 \\
\hline $\begin{array}{l}\text { Porod volume } \\
\qquad V_{p}\left(\AA^{3}\right)\end{array}$ & 252000 & 262000 & 246000 & 254000 & 264000 & 598000 & 560000 & 526000 & 578000 & 450000 \\
\hline $\begin{array}{l}M\left(V_{p} / 1.6\right) \text { in } \mathrm{kDa} \\
\text { (ratio to expected) }\end{array}$ & $158(1.1)$ & $164(1.1)$ & $154(1.1)$ & $159(1.1)$ & $165(1.1)$ & $374(1.3)$ & $350(1.2)$ & $329(1.1)$ & $361(1.2)$ & $281(1.0)$ \\
\hline
\end{tabular}

Softwares employed for SAXS data analysis

SAXS data reduction

Basic analysis: Guinier, P(r)

SVD and EFA analyses

Shape/bead modelling

Rigid body modelling

3D model representation
BM29 automated processing pipeline (BsxCuBE, ISPyBB, EDNA framework)

AUTORG, DATGNOM, GNOM, PRIMUS

BioXTas Raw

DAMMIN, DAMAVER, SUPCOMB

CORAL

CHIMERA

${ }^{\text {a }}$ Mass was estimated from Guinier and absorbance data (see also supporting information) in the following time window: for monomers, $t=1070-1100 \mathrm{~s}$; for dimers $\mathrm{t}=950-970 \mathrm{~s}$ except for \#ADC-His40 for which calculation was made from $\mathrm{t}=1035-1050 \mathrm{~s}$ to minimize contribution of oligomers, and $\mathrm{t}=930 \mathrm{~s}-950 \mathrm{~s}$ for $\mathrm{mAb}$ dimers. Expected monomeric $M$ from sequence: $145 \mathrm{kDa}$ $(\mathrm{mAb})$ and $146 \mathrm{kDa}(\mathrm{ADC})$.

b Determined with an injection volume of $20 \mu \mathrm{L}$, because the absorbance peak of the monomers was saturated by injection volumes as large as $100 \mu \mathrm{L}$. 


\section{ASSOCIATED CONTENT}

\section{Supporting Information.}

The following files are available free of charge.

SEC-SAXS analysis, mass fractals, rigid body modeling and clusters, determination of isoelectric point. (PDF)

\section{AUTHOR INFORMATION}

\section{Corresponding Author}

*dlawhine@umich.edu, christophe.tribet@ens.fr

\section{ACKNOWLEDGMENT}

This work was supported by Labex "Dynamo" ANR-11-LABX-0011-01 and a SANOFI research grant to D.L-H. We are grateful to Martha BRENNICH and Petra PERNOT for their help on the ESRF BM29 beamline and Delphine MATHIEU for revising the manuscript.

\section{ABBREVIATIONS}

ADC, Antibody-drug conjugate; mAb, Monoclonal antibody; SEC-SAXS, Size-exclusion chromatography Small-angle X-ray scattering; HMW, high molecular weight species; UPLC, Ultra-performance liquid chromatography; HPLC, High-performance liquid chromatography; AFM, Atomic force microscopy.

\section{REFERENCES}

(1) Rosenberg, A. S. Effects of Protein Aggregates: An Immunologic Perspective. AAPS J. 2006, 8 (3), E501-E507.

(2) Beck, A.; Goetsch, L.; Dumontet, C.; Corvaïa, N. Strategies and Challenges for the next 
Generation of Antibody-Drug Conjugates. Nat. Rev. Drug Discov. 2017, 16 (5), 315-337.

(3) Wakankar, A. A.; Feeney, M. B.; Rivera, J.; Chen, Y.; Kim, M.; Sharma, V. K.; Wang, Y.

J. Physicochemical Stability of the Antibody-Drug Conjugate Trastuzumab-DM1:

Changes Due to Modification and Conjugation Processes. Bioconjug. Chem. 2010, 21 (9), $1588-1595$.

(4) Buecheler, J. W.; Winzer, M.; Tonillo, J.; Weber, C.; Gieseler, H. Impact of Payload Hydrophobicity on the Stability of Antibody-Drug Conjugates. Mol. Pharm. 2018, 15 (7), 2656-2664.

(5) Panowski, S.; Bhakta, S.; Raab, H.; Polakis, P.; Junutula, J. R. Site-Specific Antibody Drug Conjugates for Cancer Therapy. MAbs 2014, 6 (1), 34-45.

(6) Shen, B. Q.; Xu, K.; Liu, L.; Raab, H.; Bhakta, S.; Kenrick, M.; Parsons-Reponte, K. L.; Tien, J.; Yu, S. F.; Mai, E.; et al. Conjugation Site Modulates the in Vivo Stability and Therapeutic Activity of Antibody-Drug Conjugates. Nat. Biotechnol. 2012, 30 (2), 184189.

(7) Frka-Petesic, B.; Zanchi, D.; Martin, N.; Carayon, S.; Huille, S.; Tribet, C. Aggregation of Antibody Drug Conjugates at Room Temperature: SAXS and Light Scattering Evidence for Colloidal Instability of a Specific Subpopulation. Langmuir 2016, 32 (19), 4848-4861.

(8) Cockrell, G. M.; Wolfe, M. S.; Wolfe, J. L.; Schöneich, C. Photoinduced Aggregation of a Model Antibody-Drug Conjugate. Mol. Pharm. 2015, 12 (6), 1784-1797.

(9) Behrens, C. R.; Ha, E. H.; Chinn, L. L.; Bowers, S.; Probst, G.; Fitch-Bruhns, M.; Monteon, J.; Valdiosera, A.; Bermudez, A.; Liao-Chan, S.; et al. Antibody-Drug 
Conjugates (ADCs) Derived from Interchain Cysteine Cross-Linking Demonstrate Improved Homogeneity and Other Pharmacological Properties over Conventional Heterogeneous ADCs. Mol. Pharm. 2015, 12 (11), 3986-3998.

(10) Tuukkanen, A. T.; Spilotros, A.; Svergun, D. I. Progress in Small-Angle Scattering from Biological Solutions at High-Brilliance Synchrotrons. IUCrJ 2017, 4, 518-528.

(11) Inouye, H.; Houde, D.; Temel, D. B.; Makowski, L. Utility of Solution X-Ray Scattering for the Development of Antibody Biopharmaceuticals. J. Pharm. Sci. 2016, 105 (11), $3278-3289$.

(12) Albertini, A. A.; Mérigoux, C.; Libersou, S.; Madiona, K.; Bressanelli, S.; Roche, S.; Lepault, J.; Melki, R.; Vachette, P.; Gaudin, Y. Characterization of Monomeric Intermediates during VSV Glycoprotein Structural Transition. PLoS Pathog. 2012, 8 (2), e1002556.

(13) Shih, O.; Yeh, Y. Q.; Liao, K. F.; Sung, T. C.; Chiang, Y. W.; Jeng, U. S. Oligomerization Process of Bcl-2 Associated X Protein Revealed from Intermediate Structures in Solution. Phys. Chem. Chem. Phys. 2017, 19 (11), 7947-7954.

(14) Volkov, V. V.; Lapuk, V. A.; Kayushina, R. L.; Shtykova, E. V.; Varlamova, E. Y.; Malfois, M.; Svergun, D. I. Low-Resolution Structure of Immunoglobulins IgG, IgM and Rheumatoid Factor IgM-RF from Solution X-Ray Scattering Data. J. Appl. Crystallogr. 2003, 36 (3 I), 503-508.

(15) Mosbaek, C. R.; Konarev, P. V.; Svergun, D. I.; Rischel, C.; Vestergaard, B. High Concentration Formulation Studies of an IgG2 Antibody Using Small Angle X-Ray 
Scattering. Pharm. Res. 2012, 29 (8), 2225-2235.

(16) Lilyestrom, W. G.; Shire, S. J.; Scherer, T. M. Influence of the Cosolute Environment on IgG Solution Structure Analyzed by Small-Angle X-Ray Scattering. J. Phys. Chem. B 2012, $116(32), 9611-9618$.

(17) Wang, W.; Lilyestrom, W. G.; Hu, Z. Y.; Scherer, T. M. Cluster Size and Quinary Structure Determine the Rheological Effects of Antibody Self-Association at High Concentrations. J. Phys. Chem. B 2018, 122 (7), 2138-2154.

(18) Fukuda, M.; Watanabe, A.; Hayasaka, A.; Muraoka, M.; Hori, Y.; Yamazaki, T.; Imaeda, Y.; Koga, A. Small-Scale Screening Method for Low-Viscosity Antibody Solutions Using Small-Angle X-Ray Scattering. Eur. J. Pharm. Biopharm. 2017, 112, 132-137.

(19) Bouchard, H.; Viskov, C.; Garcia-Echeverria, C. Antibody-Drug Conjugates - A New Wave of Cancer Drugs. Bioorganic Med. Chem. Lett. 2014, 24 (23), 5357-5363.

(20) Brennich, M. E.; Kieffer, J.; Bonamis, G.; De Maria Antolinos, A.; Hutin, S.; Pernot, P.; Round, A. Online Data Analysis at the ESRF BioSAXS Beamline, BM29. J. Appl. Crystallogr. 2016, 49, 203-212.

(21) Svergun, D. I. Determination of the Regularization Parameter in Indirect- Transform Methods Using Perceptual Criteria. J. Appl. Crystallogr. 1992, 25, 495-503.

(22) Konarev, P. V.; Volkov, V. V.; Sokolova, A. V.; Koch, M. H. J.; Svergun, D. I. PRIMUS: A Windows PC-Based System for Small-Angle Scattering Data Analysis. J. Appl. Crystallogr. 2003, 36 (5), 1277-1282.

(23) Nielsen, S. S.; Toft, K. N.; Snakenborg, D.; Jeppesen, M. G.; Jacobsen, J. K.; Vestergaard, 
B.; Kutter, J. P.; Arleth, L. BioXTAS RAW, a Software Program for High-Throughput Automated Small-Angle X-Ray Scattering Data Reduction and Preliminary Analysis. $J$. Appl. Crystallogr. 2009, 42 (5), 959-964.

(24) Svergun, D. I. Restoring Low Resolution Structure of Biological Macromolecules from Solution Scattering Using Simulated Annealing. Biophys. J. 1999, 76 (6), 2879-2886.

(25) Volkov, V.; Svergun, D. I. Uniqueness of Ab-Initio Shape Determination in Small-Angle Scattering. J. Appl. Crystallogr. 2003, 36, 860-864.

(26) Petoukhov, M. V.; Franke, D.; Shkumatov, A. V.; Tria, G.; Kikhney, A. G.; Gajda, M.; Gorba, C.; Mertens, H. D. T.; Konarev, P. V.; Svergun, D. I. New Developments in the ATSAS Program Package for Small-Angle Scattering Data Analysis. J. Appl. Crystallogr. 2012, 45 (2), 342-350.

(27) Pettersen, E. F.; Goddard, T. D.; Huang, C. C.; Couch, G. S.; Greenblatt, D. M.; Meng, E. C.; Ferrin, T. E. UCSF Chimera - A Visualization System for Exploratory Research and Analysis. J. Comput. Chem. 2004, 25 (13), 1605-1612.

(28) Kozin, M. B.; Svergun, D. I. Automated Matching of High- and Low-Resolution Structural Models. J. Appl. Crystallogr. 2001, 34, 33-41.

(29) Durand, D.; Vivès, C.; Cannella, D.; Pérez, J.; Pebay-Peyroula, E.; Vachette, P.; Fieschi, F. NADPH Oxidase Activator P67(Phox) Behaves in Solution as a Multidomain Protein with Semi-Flexible Linkers. J. Struct. Biol. 2010, 169 (1), 45-53.

(30) Receveur-Brechot, V.; Durand, D. How Random Are Intrinsically Disordered Proteins? A Small Angle Scattering Perspective. Curr. Protein Pept. Sci. 2012, 13 (1), 55-75. 
(31) Plath, F.; Ringler, P.; Graff-Meyer, A.; Stahlberg, H.; Lauer, M. E.; Rufer, A. C.; Graewert, M. A.; Svergun, D.; Gellermann, G.; Finkler, C.; et al. Characterization of MAb Dimers Reveals Predominant Dimer Forms Common in Therapeutic MAbs. MAbs 2016, 8 (5), 928-940.

(32) De Thier, P.; Bacharouche, J.; Duval, J. F. L.; Skali-Lami, S.; Francius, G. Atomic Force Microscopy Analysis of IgG Films at Hydrophobic Surfaces: A Promising Method to Probe IgG Orientations and Optimize ELISA Tests Performance. Biochim. Biophys. Acta Proteins Proteomics 2015, 1854 (2), 138-145.

(33) Chaves, R. C.; Teulon, J. M.; Odorico, M.; Parot, P.; Chen, S. W. W.; Pellequer, J. L. Conformational Dynamics of Individual Antibodies Using Computational Docking and AFM. J. Mol. Recognit. 2013, 26 (11), 596-604.

(34) Tian, X.; Langkilde, A. E.; Thorolfsson, M.; Rasmussen, H. B.; Vestergaard, B. SmallAngle X-Ray Scattering Screening Complements Conventional Biophysical Analysis: Comparative Structural and Biophysical Analysis of Monoclonal Antibodies IgG1, IgG2, and IgG4. J. Pharm. Sci. 2014, 103 (6), 1701-1710.

(35) Tian, X.; Vestergaard, B.; Thorolfsson, M.; Yang, Z.; Rasmussen, H. B.; Langkilde, A. E. In-Depth Analysis of Subclass-Specific Conformational Preferences of IgG Antibodies. IUCrJ 2015, 2, 9-18. 